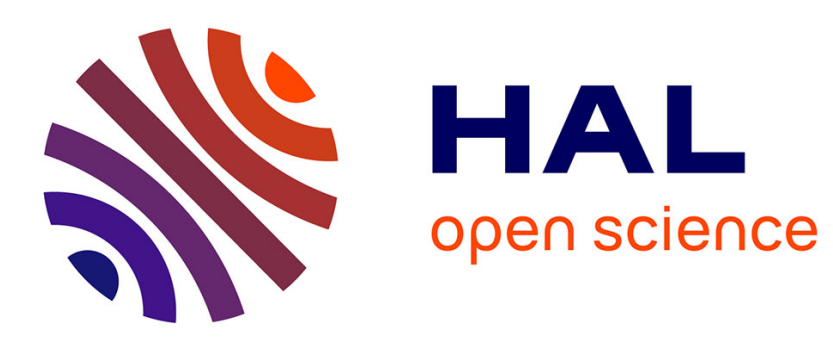

\title{
Application of a Relative Visual Performance Model in a Virtual Reality Immersive System
}

Benoit Perroud, Stephane Regnier, Andras Kemeny, Frederic Merienne

\section{To cite this version:}

Benoit Perroud, Stephane Regnier, Andras Kemeny, Frederic Merienne. Application of a Relative Visual Performance Model in a Virtual Reality Immersive System. IEEE Transactions on Visualization and Computer Graphics, 2020, 26 (10), pp.3128-3132. 10.1109/tvcg.2019.2909881 . hal-02993208

\section{HAL Id: hal-02993208 \\ https://hal.science/hal-02993208}

Submitted on 6 Nov 2020

HAL is a multi-disciplinary open access archive for the deposit and dissemination of scientific research documents, whether they are published or not. The documents may come from teaching and research institutions in France or abroad, or from public or private research centers.
L'archive ouverte pluridisciplinaire HAL, est destinée au dépôt et à la diffusion de documents scientifiques de niveau recherche, publiés ou non, émanant des établissements d'enseignement et de recherche français ou étrangers, des laboratoires publics ou privés. 


\section{Application of a Relative Visual Performance Model in a Virtual Reality Immersive System}

\author{
Benoit Perroud ${ }^{\circledR}$, Stéphane Régnier ${ }^{\circledR}$, Andras Kemeny, \\ and Frédéric Mérienne ${ }^{\circledR}$
}

\begin{abstract}
As part of an evaluation process of user experience realism in a Virtual Reality (VR) system, we focus in this paper on one of the core characteristics of vision: the relationship between contrast and luminance. The experiment aims at validating in VR reaction time predictions given by Rea and Ouellette's model. The subjects have to distinguish, as fast as they can, a target object from an uniform background. Our results did not match the predictions of the model. Our subjects showed higher performance in performing the task than expected. At low level of contrast, our subjects could easily perceive a target they should not have been able to see at all. This is explained by the size of the visual field surrounding the target: at low level of visibility, the larger the surrounding, the easier perception the is. We conclude that the Rea and Ouellette's model could be applied in VR if a specific visual field size factor was added.
\end{abstract}

Index Terms-Contrast, luminance, visual performance, virtual reality

\section{$\uparrow$}

\section{INTRODUCTION}

SIMULATION, through the use of simulators and immersive display systems, becomes an increasingly preponderant factor in the development of new products. In the automotive industry, it is largely used for relative and, sometimes, absolute validation of vehicle systems designs, such as Human Machine Interface (HMI) and Advanced Driver Assistance Systems (ADAS), or human factors studies [1]. Manufacturers hence need trustful tools in order to make decisions.

The display part of a simulator must be treated with great care and the measure of its quality appears to be essential (as an example, for driving simulators, $90 \%$ of the information used by a driver is considered to be visual, though it is yet to be fully proven numerically [2]). Our goal is to make the simulator achieve one of the five definitions of realism from [3]: the information provided to the sensory system of the body (in our case, the eyes) are to be the same that one could have in a real life situation.

This paper focuses on one of the core characteristics of vision: the relationship between contrast and luminance. These two attributes are closely linked and are key factors in the level of trust of a display. The aim is to be able to describe how much contrast and luminance one needs in Virtual Reality in order for the visual system to work as it would in a similar situaton in real life, as well as to determine what is their relationship and how the human visual system behaves at different levels of contrast and luminance.

- B. Perroud and A. Kemeny are with the Le2i (FRE 2005) Lab, Arts et Métiers, CNRS, Univ. Bourgogne Franche-Comté, HeSam, Institut Image, 2 rue T. Dumorey, Chalon-sur-Saone 71100, France, and also with the VR and Immersive Simulation Center, Renault, Guyancourt 78280, France.E-mail: b.perroud@live.fr, andras.kemeny@renault.com.

- S. Régnier is with the VR and Immersive Simulation Center, Renault, Guyancourt 78280, France.E-mail: stephane.regnier@renault.com.

- F. Mérienne is with the Le2i (FRE 2005) lab, Arts et Métiers, CNRS, Univ. Bourgogne Franche-Comté, HeSam, Institut Image, 2 rue T. Dumorey, Chalon-sur-Saone 71100 France.E-mail:frederic.merienne@ensam.eu.

\section{Related Works}

On the one hand, contrast can be roughly described as a ratio between different luminances. Its behavior is most of the time associated with a threshold above which a person is able to distinguish a target from a background and below which the same person cannot distinguish the target. Although the generally accepted threshold value is 1 percent of the background luminance, there exists numerous methods to mesure the value of the threshold and many applications [4].

On the other hand, luminance is described in photometry as the luminous intensity emerging from a surface and converging to the eye. Based on its value, it can belong to the photopic field ( $L<$ $\left.0.001 \mathrm{~cd} / \mathrm{m}^{2}\right)$, the scotopic field $\left(L>10 \mathrm{~cd} / \mathrm{m}^{2}\right)$ or the mesopic field $\left(0.001<L<10 \mathrm{~cd} / \mathrm{m}^{2}\right)$.

Rose [5], in 1948, published one of the first works that tried to evaluate performance on an absolute scale. Rose tied contrast, luminance and size in a single formula Eq. (1) where $\alpha$ is the size of the object to be seen, $L$ the luminance of the object and $C$ the contrast of the object with respect to the background. $k$ is a constant member that can be computed with other parameters.

$$
\alpha \times L \times C^{2}=k .
$$

While the Rose model gives a very good approximation of a Bayesian ideal observer, it has a limited range of validity. Hence, Burgess [6] revisited it to serve image quality purposes. Progress has been made on the model in limiting the performance by the noise of the input signal.

Though, as it appears that there exists a contrast value beyond which performance diminishes, the single threshold contrast value seems to not be enough. Following this idea, the International Commission for Eclairage (ICE), mostly based on the work of H. R. Blackwell [7], designed a model of visual performance. The approach specifies a level of performance in completing a task based on its lighting parameters such as luminance and contrast. It thus serves to establish task lighting standards. The model first computes a level of visibility at which the task is performed and then rates the overall performance. The entry parameters of the model are the difficulty of the task, the age of the observer and the lighting parameters. Its range is quite consequent as it takes in a luminance from 1 to $10000 \mathrm{~cd} / \mathrm{m}^{2}$. However, it only works for eccentricities lesser than 3 degrees. One main drawback of the model is that it should not be used in interior lighting as light is then mostly directional and specular.

However, the CIE model is only an enhanced contrast threshold model: suprathreshold values are obtained through multiplying the threshold by a constant (the visibility level). That's why Rea [8], [9] proposed a bit later his own model of visual performance (RVP). His model provides results similar to other studies measuring suprathreshold behavior. Rea's goals were to build a suprathreshold visual performance model that would be independent of task performance and consistent with the literature. His model is based on the compression effect that was theorized by Naka and Rushton in 1966 and Lipetz in 1969 as a self-shunting mechanism. The compression effect implies that at some point, despite increasing the magnitude of a stimulus, the sensation magnitude will not increase. The compression effect is described in the following equation (Eq. 2) with $R$ the response, $R_{\max }$ the maximal response, $I$ the stimulus intensity, $n$ an exponent and $K$ the stimulus intensity producing half of the maximum response.

$$
\frac{R}{R_{\max }}=\frac{I^{n}}{I^{n}+K^{n}}
$$




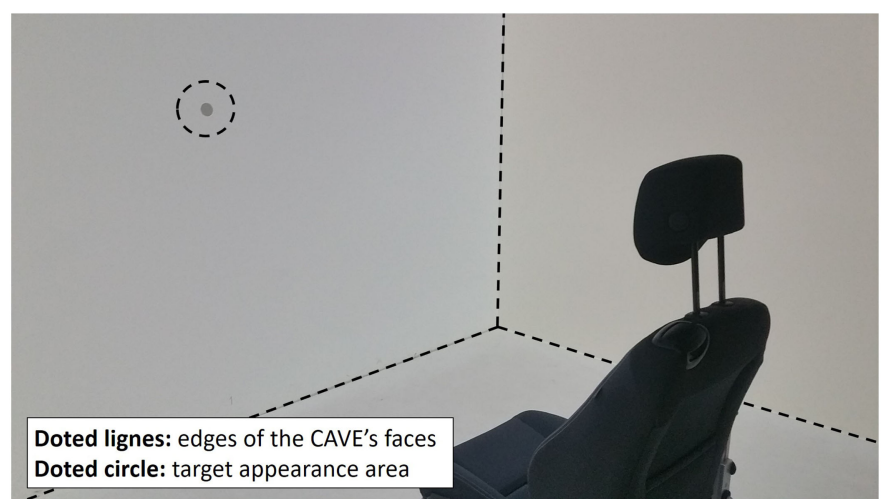

Fig. 1. View of the experimental setup. Subjects were seated 2 meters far from the front face of the CAVE-like display system and presented a disc-shaped luminous stimulus (here in black over a $(176,176,176)$ light gray background).

In a second time, Rea and Ouellette expanded Rea's RVP model with a reaction time experiment[10]. They built a model that can predict the reaction time to performing a vision task. The difference between reaction times in given conditions of luminance and contrast, and a reference reaction time, can then be linked to a RVP value. Later on, Rea and Ouellette enhanced their model to add the influence of aging [11].

Last but not least, enhancement propositions of Rea's model were made by Kambich [12] in 1991 and by O'Donell et al. 20 years later [13]. For Kambisch, though the model is a valuable tool based on robust data, the size and contrast treatments have weak theoretical bases. Kambich then replaces the sole contrast measure by three stages modeled on vision models: linear filtering, non-linear filtering and pooling stage. His model does not however consider adaptation luminance. O'Donell et al., on their side, showed that the addition of color in the model of RVP can significantly raise the performance level. Luminance and color are both important in the treatment process of the image by the brain. However, the importance of color is related to the amount of contrast: the lower, the more important color is. Under contrast values of 0.2 , color can be used to help performance, while for contrast values over 0.6 the sole luminance drives the performance.

In our study, we decided to focus on Rea and Ouellette's model, without taking into account enhancements proposed by Kambich and $\mathrm{O}^{\prime}$ Donell et al. The point is to validate the reaction time predictions, through an experimentation, that can be computed through Rea and Ouellette's model. The validation or invalidation would allow us to say whether the RVP model is fitted for Virtual Reality or not.

\section{Apparatus}

To evaluate the validity of Rea's model in Virtual Reality (VR), we came with an experimental protocol somewhat close to his. However, some hypotheses and adaptations were necessary (see below).

The experiment took place in a 4 faces CAVE-like display system and involved 32 subjects: 23 males and 9 females between the age of 20 and 27 years (mean $=25, \mathrm{sd}=1.8$ ). The CAVE-like display system specifications are as follow: a width of $3.60 \mathrm{~m}$ for a height of $2.70 \mathrm{~m}$ and a depth of $4.20 \mathrm{~m}$. The resolutions of the faces are $1600 \times 1200 p x$ for the front and floor faces, and $1920 \times 1200 p x$ for the side faces. There was one Panasonic WUXGA (85000 lumens) projector per face, equipped with an ET-DLE030 fixed focal length lens each. The projection was direct. The subjects were seated in a car seat and positioned for their eyes to be 2 meters away from the front face (Fig. 1). They were not wearing any 3D-stereoscopic glasses and the experiment was carried out with monoscopic images. They also had the instruction to keep the head still in the head-support and to focus on the location where the targets would appear.
TABLE 1

Relation between Background Color and Background Luminance - Number of Trials/Contrasts per Subject for each Background Luminance

\begin{tabular}{lcc}
\hline RGB Code & Luminance & Nbr of trials \\
\hline$(0,0,0)$ & $0.07 \mathrm{~cd} / \mathrm{m}^{2}$ & 13 \\
$(32,32,32)$ & $0.41 \mathrm{~cd} / \mathrm{m}^{2}$ & 22 \\
$(80,80,80)$ & $3.74 \mathrm{~cd} / \mathrm{m}^{2}$ & 26 \\
$(128,128,128)$ & $11.72 \mathrm{~cd} / \mathrm{m}^{2}$ & 26 \\
$(176,176,176)$ & $23.18 \mathrm{~cd} / \mathrm{m}^{2}$ & 20 \\
$(255,255,255)$ & $42.90 \mathrm{~cd} / \mathrm{m}^{2}$ & 13 \\
\hline
\end{tabular}

The whole 4 faces were displaying a unique plain grey color (the background color). No other lights were lit in the room, the CAVE-like display system screens were hence the only source of light. The background color was varying between 5 values during the experiment in order to change the amount of light arriving to the eyes of the subjects. The values and their associated luminance can be found in Table 1.

A stimulus shaped as a $2 \mathrm{~cm}$-wide (34 arcmins) disk was appearing at random timings at the center of the front face. The subjects had to press the A button on an Xbox360 controller they held in their hands as soon as they perceived the stimulus. Doing so, we could measure their reaction times. The color of the stimulus was varying over different pre-calculated levels of grey to achieve certain amounts of contrast.

In order to be able to predict what levels of gray should be used to display a specific amount of contrast for a given background luminance, we carried out a preliminary set of measures. First, all screens were lit in a solid gray. Varying the level of gray, we measured the luminance emitted. We hence could plot the transfer function linking the RGB code and the luminance. The equation was defined thanks to a least squares regression Eq. (3). Similarly, on chosen background luminances (those used in the experiment) we displayed the target and made its level of gray vary and took measures of its luminance as well. Another equation, linking the task's luminance and its RGB color code, was then extracted with a least squares regression Eq. (4). $x$ represents the RGB code value which is the same for the R, G and B components as we are only working on levels of gray. The Maple software from Maplesoft was used for the regressions. No direct calibration of the other screens was performed, only an indirect one: the side screens were lit only so that their influence was taken into account when calibrating the front screen (on which the whole experiment took place). The measures were made with a Konica-Minolta Chroma Meter CS100. All measures, and afterwards, the experiment itself, were conducted after a 1 hour warm up of the projectors as recommended by the CIE (IEC 61966-6:2005).

$$
\begin{gathered}
L_{B}(x)=-65.5 x^{4}+90.1 x^{3}+19.7 x^{2}-1.3 x+0.1 \\
L_{T}(x)=-26.1 x^{4}+38.3 x^{3}+3.7 x^{2}-0.03 x+0.02 .
\end{gathered}
$$

The contrast is originally computed in Rea's experiment like in Eq. (5). We make the hypothesis that the adaptation luminance $\left(L_{a}\right)$, which is the total luminance which arrives to the eye, is equal to the the luminance from the screens (background luminance, $L_{B}$ ). In other words, we assume that since the screens are the only source of light in the room, the adaptation luminance is the luminance emerging from the screens.

$$
C=\frac{T\left|L_{B}-L_{T}\right|}{L_{a}}
$$

Rea and Ouellette used a neural filter of transmitance $\mathrm{T}$ to pilot their contrast value: they would reduce the perceived contrast by 


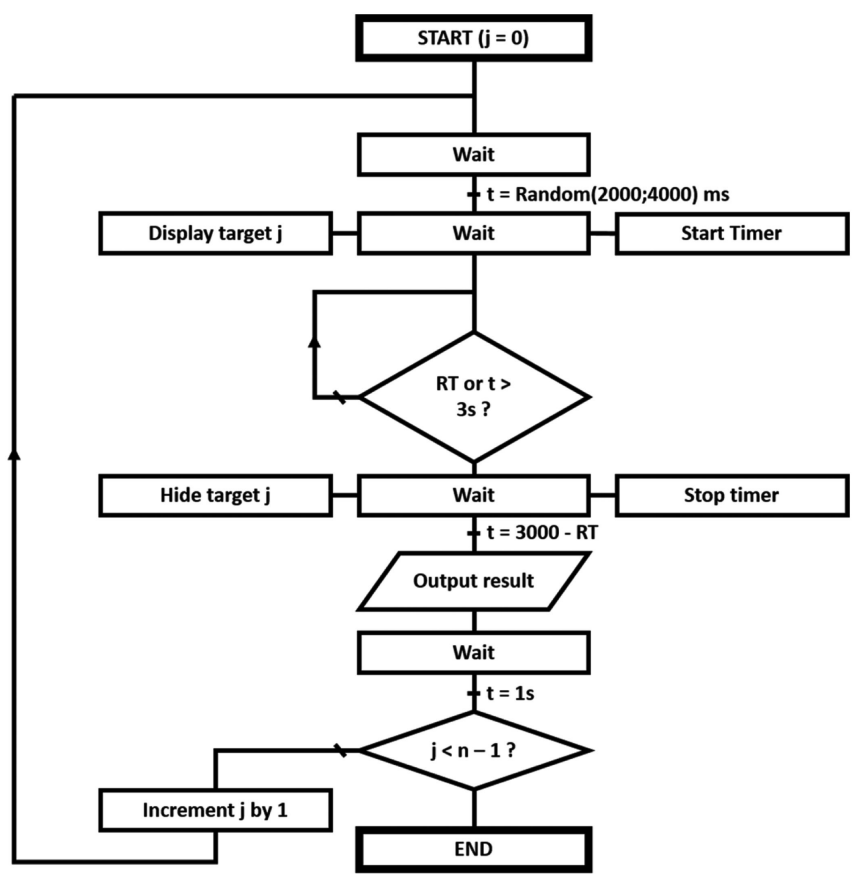

Fig. 2. Flowchart of the unfolding of a sequence of targets appearances, for a given background luminance.

densifying the neural filter in front of the eye. Plus, they used a small light directed toward the eye with a beam splitter to add on command a veiling light to reduce perceived contrast. Since we pilot our contrast directly on the screen by changing the color of the task depending of the background, we did not use any filter or veiling light and can then always set the transmitance value to $T=1$. Thanks to our hypotheses we could simplify the orignal Rea's contrast equation and define our own, suited to our experiment (Eq. (6), first member). However, Rea's equation and ours are only defined when the background is more luminous than the task $\left(L_{B} \geq L_{T}\right)$, allowing to compute contrast values by lower value only. We hence also use a slightly modified version of the equation to compute contrast values by greater value (Eq. (6), second member).

$$
C=\frac{\left|L_{B}-L_{T}\right|}{L_{B}} \text { and } C=\frac{\left|L_{B}-L_{T}\right|}{L_{T}} .
$$

\section{Method}

The subjects were first invited to sit in the car seat and then introduced to their task (stimulus detection and reaction time

TABLE 2

Theoretical Reaction Times (in ms) Predicted by the Rea and Ouellette Model

\begin{tabular}{lcccccc}
\hline $\mathrm{C} \backslash \mathrm{L}$ & 0 & 32 & 80 & 128 & 176 & 255 \\
\hline $\mathbf{0 . 0 5}$ & & & & & & \\
$\mathbf{0 . 1 0}$ & & & & & 10865 & 3594 \\
$\mathbf{0 . 1 5}$ & & & 25435 & 1924 & 1429 & 1212 \\
$\mathbf{0 . 2 0}$ & & 1686 & 1041 & 912 & 839 \\
$\mathbf{0 . 2 5}$ & & & 1039 & 788 & 725 & 685 \\
$\mathbf{0 . 3 0}$ & & & 696 & 596 & 568 & 549 \\
$\mathbf{0 . 4 0}$ & & 2663 & 577 & 517 & 499 & 546 \\
$\mathbf{0 . 5 0}$ & & 1272 & 517 & 473 & 549 & 449 \\
$\mathbf{0 . 6 0}$ & & 929 & 481 & 446 & 434 & 425 \\
$\mathbf{0 . 7 0}$ & & 773 & 456 & 426 & 416 & 409 \\
$\mathbf{0 . 8 0}$ & 4451 & 684 & 439 & 412 & 403 & 396 \\
$\mathbf{0 . 9 0}$ & 2448 & 626 & 425 & 402 & 393 & 387 \\
$\mathbf{1 . 0 0}$ & 1720 & 585 & & & & \\
\hline
\end{tabular}

TABLE 3

Measured Reaction Times (in ms) and their Respective Standard-Deviation, First Three Background Colors (0, 32 and 80)

\begin{tabular}{lccc}
\hline $\mathrm{C} \backslash \mathrm{L}$ & 0 & 32 & 80 \\
\hline $\mathbf{0 . 0 5}$ & $465 \pm 58$ & $2013 \pm 429$ & $670 \pm 112$ \\
$\mathbf{0 . 1 0}$ & $474 \pm 98$ & $663 \pm 447$ & $450 \pm 86$ \\
$\mathbf{0 . 1 5}$ & $474 \pm 85$ & $553 \pm 286$ & $439 \pm 78$ \\
$\mathbf{0 . 2 0}$ & $468 \pm 67$ & $531 \pm 323$ & $418 \pm 48$ \\
$\mathbf{0 . 2 5}$ & $465 \pm 158$ & $479 \pm 63$ & $415 \pm 68$ \\
$\mathbf{0 . 3 0}$ & $475 \pm 141$ & $507 \pm 215$ & $423 \pm 73$ \\
$\mathbf{0 . 4 0}$ & $443 \pm 74$ & $457 \pm 59$ & $451 \pm 221$ \\
$\mathbf{0 . 5 0}$ & $440 \pm 44$ & $461 \pm 78$ & $418 \pm 71$ \\
$\mathbf{0 . 6 0}$ & $440 \pm 58$ & $451 \pm 66$ & $405 \pm 52$ \\
$\mathbf{0 . 7 0}$ & $466 \pm 94$ & $450 \pm 80$ & $416 \pm 72$ \\
$\mathbf{0 . 8 0}$ & $432 \pm 68$ & $443 \pm 67$ & $402 \pm 46$ \\
$\mathbf{0 . 9 0}$ & $464 \pm 79$ & $440 \pm 61$ & $404 \pm 58$ \\
$\mathbf{1 . 0 0}$ & $448 \pm 152$ & $426 \pm 41$ & $403 \pm 52$ \\
\hline
\end{tabular}

measurement). The experiment was unfolding as follow: a background luminance was set and the subjects had to wait for 3 to 5 minutes so that their eyes would accommodate to the new luminance. When the subjects felt ready, they could launch the apparearance of the targets by pressing another button on the controller. False-positives were not taken into account (nothing occured if the subjects were to press the button when no target was displayed). When the sequence was launched, targets appeared successively with a random time between each. The target contrast relative to the background were specifically chosen to produce a desired contrast among 0.05, 0.10, 0.15, 0.20, 0.25, 0.30, 0.40, 0.50, $0.60,0.70,0.80,0.90$ and 1 . The contrast values were achieved, when possible, by target color brighter and darker than the background. Hence, the number of trial per luminance was different as for some background luminances some contrast values (either by brighter or darker value) were not possible. The order of contrast appearance and background order was randomly sorted for every subject. This helped limit the possible training effect among the subjects. When finishing a sequence at a given background luminance, the background luminance was then changed to another one and the whole process was repeated. Similarly, the order of background luminance was randomly chosen for every subject. The subjects got 6 non-measured trials before the beginning of the experiment to get used to it. They were done at a luminance that would not be used during the normal experiment. This also helped counter the training effect as there was no discovery effect during the recorded parts of the experimentation.

For a better understanding, a flowchart of the unfolding of the experiment can be found in Fig. 2. For each of the 6 background luminances (Table 1), there is a different number of displayable contrasts values (Table 1 ).

\section{Results}

To compare theoretical and measured reaction times, we first computed the predictions of Rea and Ouellette's model. Theses values can be found in Table 2. The values out of the range of the model (which predicts that one should not be able to distinguish the target over the background and hence does not provide a reaction time value) are left blank.

Some theoretical values such as $25432 \mathrm{~ms}$ or $10865 \mathrm{~ms}$ of predicted reaction time can seem rather important. Those come from a division in the model where the divider is very close to 0 (at least much closer than in the other cases) and hence the result races up very quickly.

The averaged measured reaction times values of the 32 subjects are listed in Tables 3 and 4 with their corresponding standard deviations. Since the population is over 30 subjects, the Pearson standard deviation was used. 
TABLE 4

Measured Reaction Times (in ms) and their Respective Standard-Deviation, Last Three Background Colors (128, 176 and 255)

\begin{tabular}{lccc}
\hline $\mathrm{C} \backslash \mathrm{L}$ & 128 & 176 & 255 \\
\hline $\mathbf{0 . 0 5}$ & $572 \pm 314$ & $500 \pm 290$ & $450 \pm 155$ \\
$\mathbf{0 . 1 0}$ & $421 \pm 66$ & $434 \pm 71$ & $406 \pm 50$ \\
$\mathbf{0 . 1 5}$ & $421 \pm 85$ & $434 \pm 99$ & $404 \pm 48$ \\
$\mathbf{0 . 2 0}$ & $422 \pm 87$ & $407 \pm 75$ & $432 \pm 88$ \\
$\mathbf{0 . 2 5}$ & $101 \pm 50$ & $141 \pm 62$ & $398 \pm 92$ \\
$\mathbf{0 . 3 0}$ & $399 \pm 53$ & $412 \pm 63$ & $393 \pm 72$ \\
$\mathbf{0 . 4 0}$ & $400 \pm 59$ & $406 \pm 63$ & $396 \pm 52$ \\
$\mathbf{0 . 5 0}$ & $409 \pm 62$ & $394 \pm 42$ & $415 \pm 67$ \\
$\mathbf{0 . 6 0}$ & $396 \pm 52$ & $403 \pm 6$ & $402 \pm 81$ \\
$\mathbf{0 . 7 0}$ & $394 \pm 49$ & $408 \pm 57$ & $390 \pm 48$ \\
$\mathbf{0 . 8 0}$ & $405 \pm 80$ & $394 \pm 48$ & $398 \pm 67$ \\
$\mathbf{0 . 9 0}$ & $402 \pm 61$ & $400 \pm 64$ & $399 \pm 77$ \\
$\mathbf{1 . 0 0}$ & $401 \pm 77$ & $398 \pm 64$ & $371 \pm 48$ \\
\hline
\end{tabular}

Measured values range from $371 \mathrm{~ms}$ to $670 \mathrm{~ms}$ with a mean value (of the means, excluding the diverging value, see afterwards) of $437 \mathrm{~ms}$ ( $\mathrm{SD}=53 \mathrm{~ms}$ ) and a mean standard deviation of $96 \mathrm{~ms}$ (SD = $82 \mathrm{~ms})$. A particular value $(2013 \mathrm{~ms}$ for a constrast of 0.05 and a background luminance of $0.41 \mathrm{~cd} / \mathrm{m}^{2}$ ) can be highlighted.

The performance $\mathrm{R}$ is defined as the inverse of the reaction time $\mathrm{RT}: R=1 / R T$. It is used instead of the reaction time to characterize the performance of a subject. The performance hence increases when the reaction time decreases. The graphs in Figs. 3 and 4 show both theoretical and measured performances in the reaction time experiment. The $x$-axis represents the different values of contrast, clamped by definition between 0 and 1 . The $y$-axis represents the performance $\mathrm{R}$, inverse of the reaction time (in $\mathrm{ms}^{-1}$ ). The values of the predicted performance below the threshold are out of the range of the model. They are hence set to 0 which would be equivalent to an infinite reaction time and thus a lack of reaction.

We performed Pearson's correlations statistical tests on our data to determine whether there is a correlation between the theoretical predictions and our results. The outcomes of the tests are summarized in Table 5. It appears that there is a statistical correlation for five of the background luminance setups out of six. Only the darkest setup (with an all-black background) presents a p-value greater than the 0.05 threshold.

\section{Discussion}

To begin with, our subjects were always able to perceive the targets despite the model predicting the opposite. Incidentally, there exists a significant difference in behavior between theoretical predictions and our results. Predictions theorize the performance to be increasing with the contrast level. On the other side, our subjects showed a similar performance in all conditions: their reaction times were similar for all the different lighting conditions. Following the predictions of Rea and Ouellette model, our subjects should have been increasingly faster as the contrast would increase.

At the end of the day, our results only partly match with the predictions of Rea and Ouellette's model. They diverge at low level of contrast and converge toward the theoretical values for high contrast values. There are fundamental differences that exist between the original experimental setup and ours, that may explain the differences between predictions and measurements.

It appears that only the data of the first condition (grey background 0) are not correlated. All the others sets of data show a strong positive correlation with the theoretical predictions. These statistical results therefore show that there is a correlation between the two sets of data but are not enough to specify the link. Literature suggests that the size and resulting luminance of the background influences the contrast sensitivity.
Background color: 0

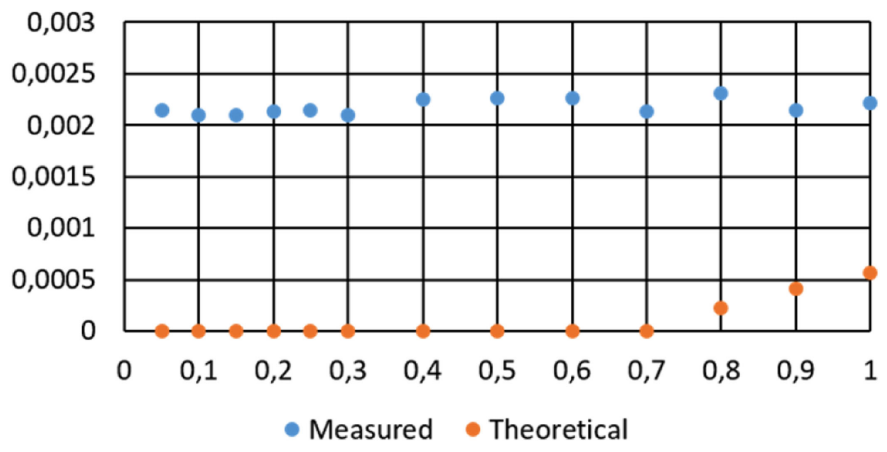

Background color: 32

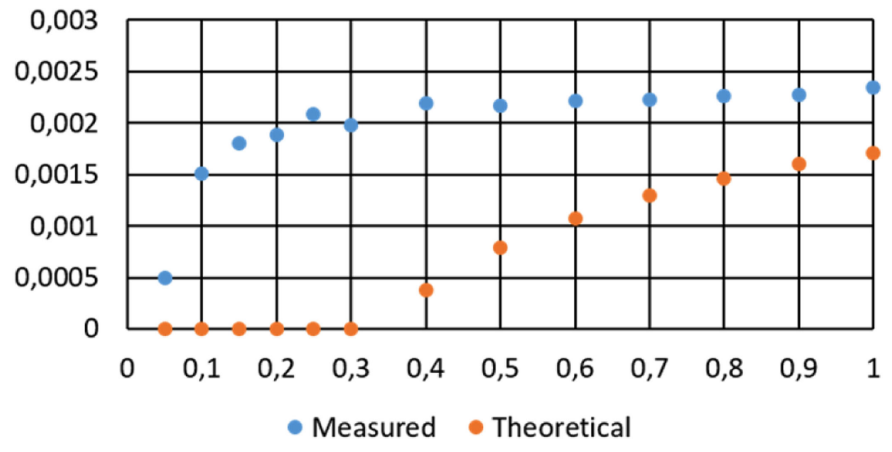

Background color: 80

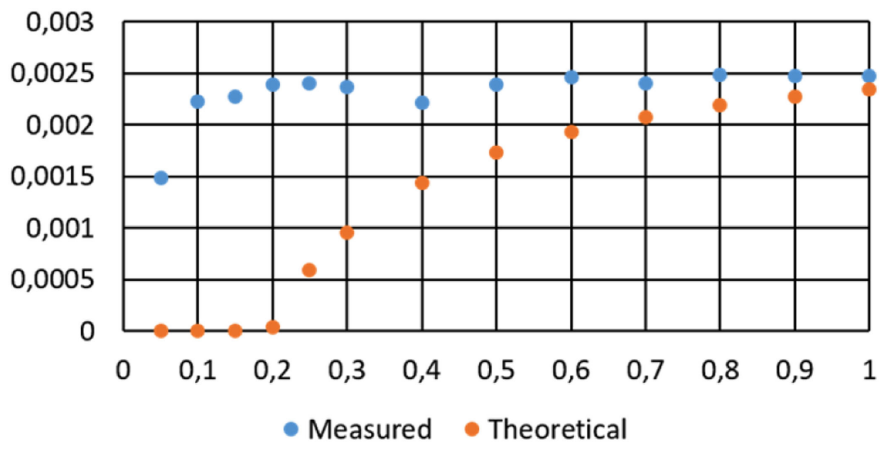

Fig. 3. Graph of the predicted performances and the real measured performances in the reaction time task for the three first background colors/luminances.

McCann et al. [14] have shown that contrast sensitivity is affected by background luminance: it changes significantly with background width and is better with a large uniform background. At the same time, results show that target visibility at the contrast threshold is significantly affected by spatial extent and luminance at the periphery of the field of view. Nevertheless, for high contrast, the influence of the immediate background size fades [15].

Hence, the more light and the more contrast displayed, the more the subjects were helped by the system to perform their task. Two behaviors coexist: on the upper side of the luminous conditions, where the measured values converge toward the theoretical ones, the subjects are correctly following the predictions of the model. On the lower side, when contrast and luminance are low, the subjects should not show such a high performance but their ability to detect the stimulus is boosted by the size of the background, which enhance the subjects' contrast sensitivity, and thus their performance. All in all, whether it is because of the background size or because of the lighting conditions, the subjects are 
Background color: 128

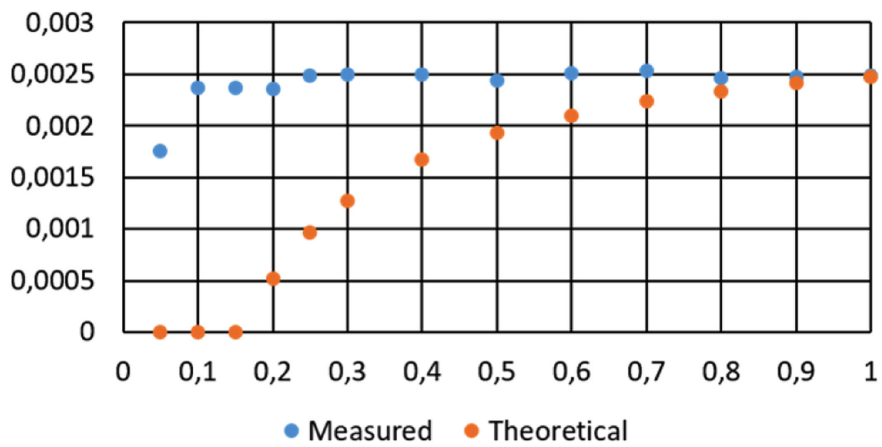

Background color: 176

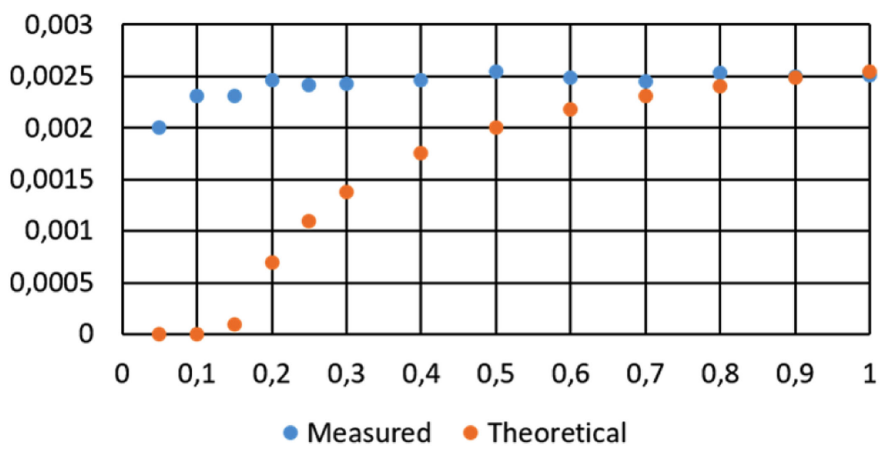

Background color: 255

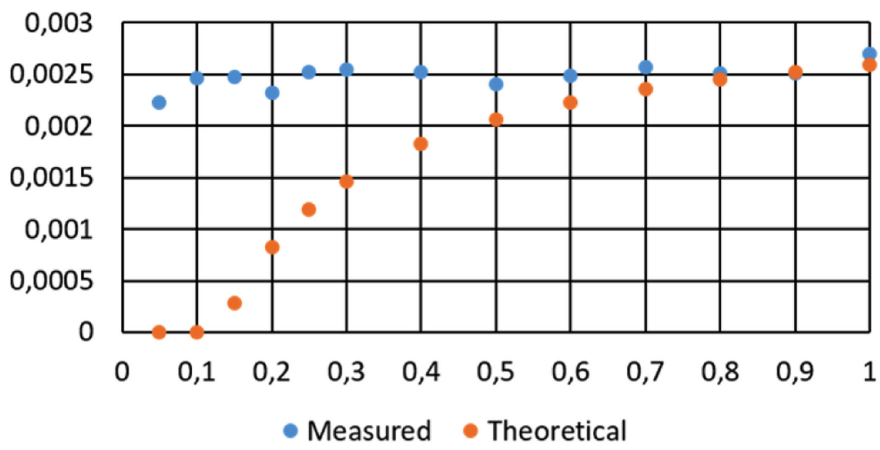

Fig. 4. Graph of the predicted performances and the real measured performances in the reaction time task for the three last background colors/luminances.

TABLE 5

Statistical Results of the Pearson's Correlation Tests

\begin{tabular}{lcccccr}
\hline $\mathrm{L}$ & 0 & 32 & 80 & 128 & 176 & 255 \\
\hline Qobs & 0.853 & 2.469 & 2.281 & 2.514 & 4.4249 & 2.715 \\
p-value & 0.412 & 0.031 & 0.043 & 0.029 & 0.001 & 0.020 \\
rhô & 0.249 & 0.597 & 0.567 & 0.604 & 0.788 & 0.634 \\
DoF & 11 & 11 & 11 & 11 & 11 & 11 \\
\hline
\end{tabular}

always in a situation in which they are able to detect the stimuli that are proposed to them. Hence, we conclude that Rea and Ouellette's model is correct but a visual field size factor must be added in the specific case of VR.

\section{Conclusion}

The aim of the study was to validate a model describing how much contrast and luminance one needs in Virtual Reality. Models already exists in the literature but none are yet applied to the specific field of Virtual Reality. The goal was to apply one of these models (Rea and Ouellette) in a VR context. An experiment similar to the one in Rea and Ouellette's papers was conducted in a CAVE-like immersive display system.

Our results show a behaviour that diverges for low contrast and brightness conditions, but converges for high conditions. However, there is a statistical correlation between the measured data and the theoretical data. The size of the background, and by extension the luminance emitted, seems to be the reason for this difference: for low contrast conditions, the larger the background on which the stimulus is displayed, the better the sensitivity of the observer. This effect disappears when the light conditions improve, which explains the convergence of the results on one side (high conditions) and the enhancement of the performance on the other (low conditions). Hence, we conclude that the Rea and Ouellette's model would be valid in Virtual Reality under the condition of an extension and the implementing of an additionnal factor that would take into account the specificities of VR such as the (visual) size of the display. We look forward to implementing such a modification factor in future work.

Finally our experiment was conducted without stereo glasses and hence does not fully represent the use of a CAVE in VR. We did not use glasses to be able to match the luminance range of Rea and Ouellette's model: glasses have an absorption level of around $66 \%$. Another experiment should be carried out to enhence our current results with the effect of stereoscopy on contrast perception.

\section{REFERENCES}

[1] A. Kemeny, "Driving simulation for virtual testing and perception studies," in Proc. DSC, 2009, pp. 15-23.

[2] M. Sivak, "The information that drivers see: Is it indeed 90\% visual?" Perception, vol. 25, no. 9, pp. 1081-1089, 1996. [Online]. Available: http:// pec. sagepub.com/lookup/doi/10.1068/p251081

[3] P. Fuchs, G. Moreau, and A. Berthoz, Le Traité de la Réalité Virtuelle. Volume 1. Paris, France: Les Presses de l'École des Mines, 2003.

[4] D. G. Pelli and P. Bex, " Measuring contrast sensitivity," Vis. Res., vol. 90, pp. 10-14, Sep. 2013. [Online]. Available: http://linkinghub.elsevier.com/ retrieve/pii/S0042698913001132

[5] A. Rose, "The sensitivity performance of the human eye on an absolute scale*," J. Opt. Soc. America, vol. 38, no. 2, Feb. 1948, Art. no. 196. [Online]. Available: https:/ / www.osapublishing.org/abstract.cfm?URI=josa-38-2-196

[6] A. E. Burgess, "The rose model, revisited," J. Opt. Soc. America, vol. 16, no. 3, pp. 633-646, 1999 .

[7] H. R. Blackwell, An Analytic Model for Describing the Influence of Lighting Parameters Upon Visual Performance. Paris, France: Bureau central de la CIE, 1981, no. no. 19/2.1-19/2.2.

[8] M. S. Rea, "Toward a model of visual performance: Foundations and data," J. Illuminating Eng. Soc., vol. 15, no. 2, pp. 41-57, Jul. 1986. [Online]. Available: http://www.tandfonline.com/doi/abs/10.1080/00994480.1986.10748655

[9] M. S. Rea, "Toward a model of visual performance: A review of methodologies," J. Illuminating Eng. Soc., vol. 16, no. 1, pp. 128-142, Jan. 1987. [Online]. Available: http://www.tandfonline.com/doi/abs/10.1080/ 00994480.1987.10748673

[10] M. S. Rea and M. J. Ouellette, "Visual performance using reaction times," Lighting Res. Technol., vol. 20, no. 4, pp. 139-153, Jan. 1988. [Online]. Available: http://lrt.sagepub.com/cgi/doi/10.1177/096032718802000401

[11] M. S. Rea and M. J. Ouellette, "Relative visual performance: A basis for application," Lighting Res. Technol., vol. 23, no. 3, pp. 135-144, Sep. 1991. [Online]. Available: http://lrt.sagepub.com/content/23/3/135

[12] D. Kambich, " An alternative relative visual performance model," J. Illuminating Eng. Soc., vol. 20, no. 1, pp. 19-27, Jan. 1991. [Online]. Available: http://www.tandfonline.com/doi/abs/10.1080/00994480.1991.10748918

[13] B. O'Donell, E. Colombo, and P. Boyce, "Colour information improves relative visual performance," Lighting Res. Technol., vol. 43, no. 4, pp. 423-438, Dec. 2011. [Online]. Available: http://lrt.sagepub.com/cgi/doi/10.1177/ 1477153511412448

[14] J. J. McCann and J. A. Hall, "Effects of average-luminance surrounds on the visibility of sine-wave gratings," J. Opt. Soc. Am., vol. 70, no. 2, pp. 212-219, Feb. 1980. [Online]. Available: http://www.osapublishing.org/abstract. cfm?URI=josa-70-2-212

[15] Y. Nakamura and Y. Akashi, "The effect of immediate background size on target detection," J. Illuminating Eng. Soc., vol. 32, no. 2, pp. 74-87, 2003. [Online]. Available: https://doi.org/10.1080/00994480.2003.10748417 